\title{
Curvature Estimates in Asymptotically Flat Lorentzian Manifolds
}

\author{
Felix Finster, Margarita Kraus
}

June 2003

\begin{abstract}
We consider an asymptotically flat Lorentzian manifold of dimension $(1,3)$. An inequality is derived which bounds the Riemannian curvature tensor in terms of the ADM energy in the general case with second fundamental form. The inequality quantifies in which sense the Lorentzian manifold becomes flat in the limit when the ADM energy tends to zero.
\end{abstract}

\section{Introduction}

In general relativity, space-time is modeled by a Lorentzian manifold $(N, g)$ of signature $(-+++)$. Gravity is described geometrically by Einstein's equations

$$
\overline{\operatorname{Ric}}-\frac{1}{2} \bar{s} g=-8 \pi T,
$$

where $\bar{R}$ is the curvature corresponding to the Levi-Civita connection $\bar{\nabla}$ on $N$, $\overline{\text { Ric }}$ is the Ricci curvature and $\bar{s}$ the scalar curvature. Here $T$ is the energy-momentum tensor; it tells about the distribution of matter in space-time and gives a local concept of energy and momentum. The fact that the local energy density should be positive is expressed by the dominant energy condition, saying that for each $p \in N$ and each timelike vector $u \in T_{p} N$,

$$
T_{\alpha \beta} u^{\beta} \text { is timelike and } T(u, u) \leq 0 .
$$

We choose a space-like hypersurface $M \subset N$ and let $(g, h)$ be the induced Riemannian metric and the second fundamental form on $M$, respectively. In many physical situations, matter is localized in a bounded region of space, and the gravitational field falls off at large distance from the sources. This leads to the definition of asymptotic flatness; for simplicity we consider only one asymptotic end.

Def. 1.1 $M$ is asymptotically flat if there is a compact set $K \subset M$ and a diffeomorphism $\Phi$ which maps $M \backslash K$ to the region $\mathbb{R}^{3} \backslash B_{r}(0)$ outside a ball of radius $r$. Under this diffeomorphism, the metric and second fundamental form should be of the form

$$
\begin{array}{lll}
\left(\Phi_{*} g\right)_{i j}=\delta_{i j}+\mathcal{O}\left(r^{-1}\right), & \partial_{k}\left(\Phi_{*} g\right)_{i j}=\mathcal{O}\left(r^{-2}\right), & \partial_{k} \partial_{l}\left(\Phi_{*} g\right)_{i j}=\mathcal{O}\left(r^{-3}\right) \\
\left(\Phi_{*} h\right)_{i j}=\mathcal{O}\left(r^{-2}\right), & \partial_{k}\left(\Phi_{*} h\right)_{i j}=\mathcal{O}\left(r^{-3}\right) .
\end{array}
$$

In asymptotically flat manifolds, one can introduce the ADM energy and momentum, which have the interpretation as the total energy and momentum of space-time. 
Def. 1.2 The ADM energy and momentum (E,P) are defined by

$$
\begin{aligned}
E & =\frac{1}{16 \pi} \lim _{R \rightarrow \infty} \sum_{i, j=1}^{3} \int_{S_{R}}\left(\partial_{j}\left(\Phi_{*} g\right)_{i j}-\partial_{i}\left(\Phi_{*} g\right)_{j j}\right) d \Omega^{i} \\
P_{k} & =\frac{1}{8 \pi} \lim _{R \rightarrow \infty} \sum_{i=1}^{3} \int_{S_{R}}\left(\left(\Phi_{*} h\right)_{k i}-\sum_{j=1}^{3} \delta_{k i}\left(\Phi_{*} h\right)_{j j}\right) d \Omega^{i},
\end{aligned}
$$

where $d \Omega^{i}=\nu^{i} d u$, du is the area form, and $\nu$ is the normal vector to $S_{R} \subset \mathbb{R}^{3}$.

This definition is indeed independent of the the choice of $\Phi$ [1].

It is a major problem of mathematical relativity to understand the relation between $(E, P)$ and the geometry of space-time. A particular aspect of this problem is the question whether and in which sense $E$ and $P$ control the Riemannian curvature tensor. In 4 this question was addressed in the time-symmetric case (i.e. when $h \equiv 0$ ). $L^{2}$-estimates for the Riemannian curvature tensor where derived on $M \backslash D$, where $D$ is an "exceptional set" of small volume. In [5] these estimates were generalized to higher dimensions. In the present paper we treat the physically relevant case with second fundamental form. This is our main result:

Theorem 1.3 We choose $L \geq 3$ such that

$$
\left(L^{\alpha}-1\right)^{2} \geq C \frac{4 \pi E+\|h\|_{2}}{k^{2}\left(k+24\|h\|_{3}\right)^{2}}\left\||h|^{2}+|\nabla h|\right\|_{3}
$$

where

$$
\alpha=\left(1+24 \frac{\|h\|_{3}}{k}\right)^{-1} .
$$

Then there is a set $U$ with measure bounded by

$$
\mu(U) \leq c_{1} \frac{L^{6}}{k^{2}}\left(4 \pi E+\|h\|_{2}^{2}\right)
$$

such that on $M \backslash U$ the following inequality holds,

$$
\begin{aligned}
& \int_{M \backslash U} \eta\left|\bar{R}_{M}\right|^{2} d \mu \leq c_{2} \sup _{M}\left(|\Delta \eta|+|\nabla \eta||h|+\eta\left(|R|+|h|^{2}+|\bar{\nabla} h|\right) E\right. \\
& \quad+c_{3} L \sup _{M}\left(\eta\left(\left|\bar{\nabla} R_{M}\right|+|h|\left|\bar{R}_{M}\right|\right)\right) \sqrt{E} \\
& \quad+c_{4} \frac{\sqrt{L+1}}{k}\left(\sup _{M} \eta\right) \sqrt{\left\||h|^{2}+|\nabla h|\right\|_{6 / 5}}\left\|\left|\overline{\nabla R}_{M}\right|+|h| \mid \bar{R}_{M}\right\|_{5 / 12} \sqrt{E} .
\end{aligned}
$$

Here $c_{1}, \ldots, c_{4}$ are numerical constants (independent of $L$ and the geometry), $\eta \in C^{2}(M)$ is a positive test function, $\bar{R}_{M}$ is the Riemannian curvature tensor of $N$ restricted to $M$, and $k$ is the isoperimetric constant $k=\inf A / V^{\frac{2}{3}}$.

For the proof we use Witten's solutions of the hypersurface Dirac equation [9, 8] and consider second derivatives of the spinors. In order to control the Weyl tensor, we work similar as in [5] with the spinor operator $\Pi$, which is built up of a whole family of solutions of the hypersurface Dirac equation. The presence of the second fundamental form leads to the difficulty that the function $|\Psi|^{2}$ is no longer subharmonic, making it impossible to estimate the norm of the spinor with the maximum principle. In order get around this difficulty, we first construct a barrier function $F$, which is a solution of a suitable Poisson equation. We then derive Sobolev estimates for $F$, and these finally give us control of $\left\||\Psi|^{2}-1\right\|_{L^{6}(M)}$. 


\section{Basic Facts about Spinors and the Hypersurface Dirac Operator}

In this section we recall some basic facts about spinors and the Dirac operator on Lorentzian spin manifolds; for details the reader is referred to [3, 6 .

Let $(N, g)$ be a Lorentzian spin-manifold with spin structure $Q_{N} \rightarrow N$. Let $\kappa: \operatorname{Spin}(1, n-1) \rightarrow \Delta_{1, n-1}$ denote the spinor representation and

$$
\Sigma_{N}=Q_{N} \times{ }_{\kappa} \Delta_{1, n-1}
$$

the associated spinor bundle. We denote the Clifford multiplication of a tangent vector $X$ with a spinor $\psi$ by $\mu(X, \psi)=: X \cdot \psi$. On $\Delta_{1, n-1}$ there exists an indefinite scalar product $\langle\cdot, \cdot\rangle$ of signature $(2,2)$, which is invariant under $\operatorname{Spin}^{+}(1, n-1)$ and is unique up to a constant. This inner product induces on $\Sigma_{N}$ an indefinite scalar product, which we again denote by $\langle\cdot, \cdot\rangle$. For a timelike vector field $\nu$, the inner product

$$
(\phi, \psi):=\langle\phi, \nu \cdot \psi\rangle
$$

is positive.

The scalar products $(\cdot, \cdot)$ and $\langle\cdot, \cdot\rangle$ also define scalar products on the fibres of the bundles of $k$-forms $\Lambda^{k} T^{*} N \otimes \Sigma_{N}$ and the bundle of $k$-linear mappings $\left(\otimes^{k} T^{*} N \otimes \Sigma_{N}\right)$ by

$$
(\eta, \xi)_{p}:=\sum_{i_{1}, \ldots, i_{k}}\left(\eta\left(e_{i_{1}}, \ldots, e_{i_{k}}\right), \xi\left(e_{i_{1}}, \ldots, e_{i_{k}}\right)\right)_{p}
$$

and analogously

$$
\langle\eta, \xi\rangle_{p}:=\sum_{i_{1}, \ldots, i_{k}}\left\langle\eta\left(e_{i_{1}}, \ldots, e_{i_{k}}\right), \xi\left(e_{i_{1}}, \ldots, e_{i_{k}}\right)\right\rangle
$$

where $e_{1}, \ldots, e_{n}$ is an orthonormal frame. The Levi-Civita connection on $N$ induces a covariant derivative $\bar{\nabla}$ on $\Gamma \Sigma_{N}$.

This covariant derivative is isometric with respect to $\langle\ldots\rangle$, i.e.

$$
X\langle\varphi, \psi\rangle=\left\langle\bar{\nabla}_{X} \varphi, \psi\right\rangle+\left\langle\varphi, \bar{\nabla}_{X} \psi\right\rangle
$$

for all sections $\varphi, \psi$ in $\Sigma_{N}$. Its curvature tensor $\bar{R}^{\Sigma} \in \Omega^{2}\left(N, \Sigma_{N}\right)$ is defined by

$$
\bar{R}^{\Sigma}(X, Y) \psi=\left(\bar{\nabla}^{2} \psi\right)(X, Y)-\left(\bar{\nabla}^{2} \psi\right)(Y, X),
$$

where $\left(\bar{\nabla}^{2} \psi\right)(X, Y)=\bar{\nabla}_{X} \bar{\nabla}_{Y}-\bar{\nabla}_{\bar{\nabla}_{X} Y}$. It is related to the curvature tensor $\bar{R}$ of the Lorentzian manifold $(N, g)$ by the formula

$$
\bar{R}^{\Sigma} \psi=\frac{1}{4} \sum_{\alpha, \beta=1}^{n}\left\langle\bar{R} e_{\alpha}, e_{\beta}\right\rangle e_{\alpha} \cdot e_{\beta} \cdot \psi
$$

The Dirac operator on the Lorentzian manifold $N$ is defined by the composition of the covariant derivative $\bar{\nabla}$ with the Clifford multiplication $\mu$,

$$
\bar{D}: \Gamma \Sigma_{N} \stackrel{\bar{\nabla}}{\longrightarrow} \Gamma\left(T^{*} N \otimes \Sigma_{N}\right) \stackrel{\mu}{\longrightarrow} \Gamma \Sigma_{N}
$$


where the cotangent bundle $T^{*} N$ has been identified with the tangent bundle $T N$ via the metric. In a local orthonormal frame $\left(e_{1}, \ldots, e_{n}\right)$, the Dirac operator is given by

$$
\bar{D}=\sum_{\alpha=1}^{n} e_{\alpha} \cdot \bar{\nabla}_{e_{\alpha}}
$$

We point out that, in contrast to the Riemannian case, the Dirac operator on a Lorentzian manifold is not elliptic.

In what follows, we restrict attention to the physically relevant case of a 4-dimensional Lorentzian manifold with a given 3-dimensional asymptotically flat space like hypersurface $M \subset N$. We choose a normal unit vector field $\nu$ on $M$ and consider the corresponding positive definite scalar product (2.1). We set $|\psi|=(\psi, \psi)^{\frac{1}{2}}$.

The covariant derivative $\bar{\nabla}$ is not compatible with $(\cdot, \cdot)$, but

$$
X(\varphi, \psi)=\left(\bar{\nabla}_{X} \varphi, \psi\right)+\left(\varphi, \bar{\nabla}_{X} \psi\right)+\left(\varphi, \nu \cdot \bar{\nabla}_{X} \nu \cdot \psi\right)
$$

holds for spinor fields $\varphi, \psi \in \Gamma\left(\Sigma_{N} \mid M\right)$. Using the definition of the second fundamental form

$$
h_{i j}=-\left\langle e_{i}, \bar{\nabla}_{e_{j}} \nu\right\rangle
$$

for an orthonormal frame $\left(e_{1}, e_{2}, e_{3}\right)$ on $M$, this formula can be written as

$$
e_{i}(\varphi, \psi)=\left(\bar{\nabla}_{e_{i}} \varphi, \psi\right)+\left(\varphi, \bar{\nabla}_{e_{i}} \psi\right)-h_{i j}\left(\varphi, \nu \cdot e_{j} \cdot \psi\right) .
$$

This leads us to define the adjoint of $\bar{\nabla}$ by $\bar{\nabla}_{X}^{*} \psi=-\bar{\nabla}_{X} \psi-\nu \cdot \bar{\nabla}_{X} \nu \cdot \psi$ or, in an orthonormal frame,

$$
\bar{\nabla}_{e_{i}}^{*} \psi=-\bar{\nabla}_{e_{i}} \psi+h_{i j} \nu \cdot e_{j} \cdot \psi .
$$

On a spacelike hypersurface, there exists an intrinsic Riemannian Dirac operator, but we shall not consider it here. Instead, we will only be concerned with the so called hypersurface Dirac operator $\bar{D}_{M}$,

$$
\bar{D}_{M}:=\left.\bar{D}\right|_{M}: \Gamma \Sigma_{\bar{M}}\left|M \rightarrow \Gamma \Sigma_{\bar{M}}\right| M
$$

It is the restriction of the Dirac operator of the Lorentzian manifold $N$ to $M$; more precisely,

$$
\Gamma\left(\Sigma_{\bar{M}} \mid M\right) \stackrel{\bar{\nabla}}{\longrightarrow} \Gamma\left(T^{*} M \otimes \Sigma_{\bar{M}} \mid M\right) \longrightarrow \Gamma\left(\Sigma_{\bar{M}} \mid M\right),
$$

where $\bar{\nabla}$ denotes the covariant derivative in direction $M$. According to [8], the square of the hypersurface Dirac operator satisfies the Weitzenböck formula

$$
\bar{D}_{M}^{2}=\bar{\Delta}^{s}+\Re \text {. }
$$

Here $\bar{\Delta}^{s}$ is the Laplacian $\bar{\Delta}^{s} \psi=\bar{\nabla}^{*} \bar{\nabla} \psi=\operatorname{tr}\left(-\bar{\nabla}^{2}-\nu \cdot \bar{\nabla} \nu \cdot \bar{\nabla} \psi\right)$ or, in an orthonormal frame,

$$
\bar{\Delta}^{s} \psi=-\sum_{i, j}\left(\bar{\nabla}_{e_{j}} \bar{\nabla}_{e_{j}} \psi-\bar{\nabla}_{\nabla_{e_{j}} e_{j}} \psi-h_{i j} \nu \cdot e_{i} \cdot \bar{\nabla}_{e_{j}} \psi\right)
$$

and $\Re$ is the curvature expression $\Re=\frac{1}{4}\left(\bar{s}+2 \overline{\operatorname{Ric}}(\nu, \nu)+2 \sum_{i=1}^{3} \overline{\operatorname{Ric}}\left(\nu, e_{i}\right)\left(\nu \cdot e_{i}\right)\right.$. The dominant energy condition (1.1) yields that $\Re \geq 0$. 
In the coordinates induced by the diffeomorphism $\phi$ of Definition 1.1, we choose a constant spinor $\psi_{0}$ of norm one in the asymptotic end and consider the boundary value problem

$$
\bar{D}_{M} \psi=0, \quad \lim _{|x| \rightarrow \infty} \psi(x)=\psi_{0} \text { with }\left|\psi_{0}\right|=1 .
$$

The existence and uniqueness of a solution of (2.5) is proven in [8]. The solution decays at infinity as

$$
\psi=\psi_{0}+\mathcal{O}\left(r^{-1}\right), \partial_{j} \psi=\mathcal{O}\left(r^{-2}\right), \partial_{k l} \psi=\mathcal{O}\left(r^{-3}\right) .
$$

Using the Weitzenböck formula (2.4), it is shown in [8] that for a solution of (2.5),

$$
\|\nabla \psi\|_{L^{2}(M)}^{2}=4 \pi\left(E\left|\psi_{0}\right|^{2}+\left(\psi_{0}, P \cdot \psi_{0}\right)\right)-(\psi, \Re \psi) \leq 4 \pi\left(E+\left(\psi_{0}, P \cdot \psi_{0}\right)\right),
$$

where $P=P_{k} \cdot e_{k}$ is the momentum as defined by (1.2). If we choose $\psi_{0}$ such that $\left(\psi_{0} \mid P \cdot \psi_{0}\right)=-|P|$, we obtain the positive mass theorem 9 , 8 ]

$$
0 \leq 4 \pi(E-|P|)
$$

For general $\psi_{0},(2.6)$ and (2.7) give rise to an $L^{2}$-bound of $\nabla \psi$,

$$
\|\nabla \psi\|_{L^{2}(M)}^{2} \leq 4 \pi(E+|P|) \leq 8 \pi E .
$$

\section{A-priori Estimates for Harmonic Spinors}

In what follows, we let $\psi \in \Gamma \Sigma_{N} \mid M$ be a solution of the boundary value problem (2.5). We refer to $\psi$ as a harmonic spinor. We begin by deriving an upper bound for the measure of the set where a harmonic spinor is large. For any $L \geq 1$, we introduce the set $\Omega_{L}=\Omega_{L}(\psi)$ by

$$
\Omega_{L}(\psi)=\{x \in M:|\psi(x)| \geq L\} .
$$

Lemma 3.1 For any harmonic spinor $\psi$ and every $L \geq 1$, the volume of $\Omega_{L}$ is bounded by

$$
\mu\left(\Omega_{L}\right)^{\frac{1}{3}} \leq \frac{192}{\left(L^{\alpha}-1\right)^{2}} \frac{4 \pi E+\|h\|_{2}^{2}}{k^{2}},
$$

where the exponent $\alpha$ is

$$
\alpha=\left(1+24 \frac{\|h\|_{3}}{k}\right)^{-1} .
$$

The proof uses the the following Sobolev inequality, which is derived in [5].

Lemma 3.2 Let $M$ be an asymptotically flat manifold of dimension $n \geq 3$. Then every non-negative function $g \in C^{\infty}(M) \cap H^{1,2}(M)$ with $\lim _{|x| \rightarrow \infty} g(x)=0$ satisfies the inequality

$$
\|g\|_{q} \leq \frac{q}{k}\|\nabla g\|_{2} \quad \text { with } \quad q=\frac{2 n}{n-2}
$$

and $k$ the isoperimetric constant. 
Proof of Lemma [3.1. Applying the Schwarz inequality in (2.3), we obtain for every $\alpha \in \mathbb{R}$,

$$
\left.\left.|\nabla| \psi\right|^{\alpha}|\leq \alpha| \psi\right|^{\alpha-2}\left(|\nabla \psi||\psi|+|h||\psi|^{2}\right) .
$$

We take the square and use the inequality $(x+y)^{2} \leq 2\left(x^{2}+y^{2}\right)$,

$$
\left.\left.|\nabla| \psi\right|^{\alpha}\right|^{2} \leq 2 \alpha^{2}\left(|\nabla \psi|^{2}|\psi|^{2 \alpha-2}+|h|^{2}|\psi|^{2 \alpha}\right) .
$$

Choosing $x \in \Omega_{L}$ and $\alpha \in(0,1]$, the factor $|\psi(x)|^{2 \alpha-2}<1$, and thus at $x$,

$$
\left.\left.|\nabla| \psi\right|^{\alpha}\right|^{2} \leq 2 \alpha^{2}\left(|\nabla \psi|^{2}+|h|^{2}|\psi|^{2 \alpha}\right)
$$

We integrate over $\Omega_{L}$ and apply Lemma 3.2 as well as (2.8),

$$
\left\||\psi|^{\alpha}-1\right\|_{L^{6}\left(\Omega_{L}\right)}^{2} \leq \frac{72 \alpha^{2}}{k^{2}}\left(8 \pi E+\left\||h|^{2}|\psi|^{2 \alpha}\right\|_{L^{1}\left(\Omega_{L}\right)}\right) .
$$

The last inequality has the disadvantage that the spinor also appears on the right. Therefore, we apply the inequality $|\psi|^{2 \alpha} \leq 2\left(|\psi|^{\alpha}-1\right)^{2}+2$ and Hölder to obtain

$$
\left\||\psi|^{\alpha}-1\right\|_{L^{6}\left(\Omega_{L}\right)}^{2} \leq \frac{72 \alpha^{2}}{k^{2}}\left(8 \pi E+2\|h\|_{3}^{2}\left\|\left(|\psi|^{\alpha}-1\right)\right\|_{L^{6}\left(\Omega^{1}\right)}^{2}+2\|h\|_{2}^{2}\right) .
$$

Now we can combine the terms involving the spinors,

$$
\left[1-\frac{12^{2} \alpha^{2}}{k^{2}}\|h\|_{3}^{2}\right]\left\||\psi|^{\alpha}-1\right\|_{L^{6}\left(\Omega_{L}\right)}^{2} \leq \frac{12^{2} \alpha^{2}}{k^{2}}\left(4 \pi E+\|h\|_{2}^{2}\right) .
$$

We choose $\alpha$ according to (3.2). Then the second term in the square brackets in (3.3) is bounded by

$$
\frac{12^{2} \alpha^{2}}{k^{2}}\|h\|_{3}^{2} \leq \frac{1}{4}
$$

and thus

$$
\left\||\psi|^{\alpha}-1\right\|_{L^{6}\left(\Omega_{L}\right)}^{2} \leq 192 \frac{\alpha^{2}}{k^{2}}\left(4 \pi E+\|h\|_{2}^{2}\right) .
$$

We finally apply the estimate

$$
\mu\left(\Omega_{L}\right)^{\frac{1}{3}} \leq \frac{1}{\left(L^{\alpha}-1\right)^{2}}\left\||\psi|^{\alpha}-1\right\|_{L^{6}\left(\Omega_{L}\right)}^{2} .
$$

In the time-symmetric case, Lemma 3.1 reduces to the inequality

$$
\mu\left(\Omega_{L}\right)^{\frac{1}{3}} \leq \frac{192}{(L-1)^{2}} \frac{4 \pi E}{k^{2}}
$$

showing that for large $L, \mu\left(\Omega_{L}\right)$ decays at least $\sim L^{-6}$. On the other hand, it was shown in the time-symmetric case [4] that the function $|\psi|^{2}$ is subharmonic, and thus the maximum principle gave the bound

$$
|\psi|^{2} \leq 1
$$

This shows that if $h \equiv 0, \mu\left(\Omega_{L}\right)$ is indeed zero for all $L>1$. We conclude that the estimate (3.4) is certainly not optimal if $h \equiv 0$. We shall now improve Lemma 3.2 such 
that in the time-symmetric case we recover (3.5). We let $\left(e_{1}, e_{2}, e_{3}\right)$ be an orthonormal frame in a neighborhood of $x$ with $\left(\bar{\nabla}_{i} e_{j}\right)(x)=0$. Then the Laplacian of $|\psi|^{2}$ at $x$ is computed as follows,

$$
\begin{aligned}
\Delta|\psi|^{2}= & \sum_{j=1}^{3} \partial_{j}\left(\left(\bar{\nabla}_{j} \psi, \psi\right)+\left(\psi, \bar{\nabla}_{j} \psi\right)+\left(\psi, \nu \cdot \bar{\nabla}_{j} \nu \cdot \psi\right)\right) \\
= & 2|\bar{\nabla} \psi|^{2}+2 \operatorname{Re}\left(\bar{\nabla}_{j} \bar{\nabla}_{j} \psi, \psi\right)+2\left(\bar{\nabla}_{j} \psi, \nu \cdot \bar{\nabla}_{j} \nu \cdot \psi\right) \\
& +\left(\psi, \nu \cdot \bar{\nabla}_{j} \nu \cdot \bar{\nabla}_{j} \psi\right)+\left(\psi, \bar{\nabla}_{j}\left(\nu \cdot \bar{\nabla}_{j} \nu \cdot \psi\right)\right)-\left|\bar{\nabla}_{j} \nu\right|^{2}|\psi|^{2} \\
= & \left.2|\bar{\nabla} \psi|^{2}-2 \operatorname{Re}\left(\bar{\nabla}^{*} \bar{\nabla} \psi, \psi\right)+2 \operatorname{Re}\left(\bar{\nabla}_{j} \psi, \nu \cdot \bar{\nabla}_{j} \nu \cdot \psi\right)+\left(\psi, \nu \cdot \bar{\nabla}_{j, j}^{2} \nu \cdot \psi\right)\right)
\end{aligned}
$$

Using the Weitzenböck formula, we obtain for a harmonic spinor the inequality

$$
\left.\left.|\Delta| \psi\right|^{2}|\geq 2 \operatorname{Re}(\Re \psi, \psi)+2| \bar{\nabla} \psi\right|^{2}-2|\bar{\nabla} \psi||\psi||\bar{\nabla} \nu|-|\psi|^{2} \cdot\left(\sum_{j=1}^{3}\left|\bar{\nabla}_{j, j}^{2} \nu\right|\right),
$$

where we set

$$
|\bar{\nabla} \nu|^{2}=\sum_{i, j} h_{i j}^{2} \quad \text { and } \quad\left|\bar{\nabla}_{j, j}^{2} \nu\right|=3 \sqrt{\sum_{k}\left(\partial^{j} h_{j k}\right)^{2}} .
$$

Using the short notation

$$
|h|:=|\bar{\nabla} \nu| \quad \text { and } \quad|\nabla h|:=\sum_{j=1}^{3}\left|\bar{\nabla}_{j, j}^{2} \nu\right|,
$$

we can write the last inequality in the compact form

$$
\begin{aligned}
\Delta|\psi|^{2} & \geq 2 \operatorname{Re}(\Re \psi, \psi)+2|\bar{\nabla} \psi|^{2}-2|\bar{\nabla} \psi||\psi||h|-|\psi|^{2}|\nabla h| \\
& \geq 2 \operatorname{Re}(\Re \psi, \psi)-\left(\frac{1}{2}|h|^{2}+|\nabla h|\right)|\psi|^{2} .
\end{aligned}
$$

In the special case $h \equiv 0$, we recover that $|\psi|^{2}$ is subharmonic, and the maximum principle gives (3.5). Our method for treating the general case is to construct a barrier function $F$ by solving the Poisson equation and to estimate $F$ using Sobolev techniques and the volume bound of Lemma 3.1 .

Proposition 3.3 Suppose that $L>1$ is chosen so large that

$$
\left(L^{\alpha}-1\right)^{2} \geq C \frac{4 \pi E+\|h\|_{2}}{k^{2}\left(k+24\|h\|_{3}\right)^{2}}\left\||h|^{2}+|\nabla h|\right\|_{3}
$$

with $\alpha$ as in Lemma 3.1 and $C=6 \cdot 48^{2}$ a numerical constant. Then the harmonic spinor $\psi$ is bounded on $\Omega_{L}$ by

$$
\left\||\psi|^{2}-1\right\|_{L^{6}\left(\Omega_{L}\right)} \leq \frac{72}{k^{2}}(L+1)\left\||h|^{2}+|\nabla h|\right\|_{L^{6 / 5}} .
$$


Proof. We set $\rho=-\left(|h|^{2}+|\nabla h|\right)$ and let $g$ be the solution of the Poisson equation $\Delta g=\rho|\psi|^{2}$ with boundary conditions $\lim _{x \rightarrow \infty} g(x)=0$ (For the existence of this solution see 2. Theorem1.7]). Then $\Delta\left(|\psi|^{2}-g\right) \geq 0$, and the maximum principle yields that

$$
|\psi|^{2} \leq 1+g .
$$

The Sobolev inequality of Lemma 3.2. Gauss' theorem, and the Hölder inequality give

$$
\|g\|_{6}^{2} \leq \frac{36}{k^{2}}\|\nabla g\|_{2}^{2}=\left.\frac{36}{k^{2}} \int_{M}\left|\rho\left\|\left.\psi\right|^{2} g d M \leq \frac{36}{k^{2}}\right\| \rho\right| \psi\right|^{2}\left\|_{\frac{6}{5}}\right\| g \|_{6}
$$

and thus

$$
\|g\|_{6} \leq \frac{36}{k^{2}}\left\|\rho|\psi|^{2}\right\|_{\frac{6}{5}} .
$$

Combining (3.7) and (3.8), we obtain for any $L \geq 1$,

$$
\begin{aligned}
\left\||\psi|^{2}-1\right\|_{L^{6}\left(\Omega_{L}\right)} & \leq\|g\|_{L^{6}\left(\Omega_{L}\right)} \leq \frac{36}{k^{2}}\left\|\rho|\psi|^{2}\right\|_{\frac{6}{5}} \\
& \leq \frac{36}{k^{2}}\left(L\|\rho\|_{L^{6 / 5}\left(M \backslash \Omega_{L}\right)}+\left\|\rho|\psi|^{2}\right\|_{L^{6 / 5}\left(\Omega_{L}\right)}\right) \\
& \leq \frac{36}{k^{2}}\left(L\|\rho\|_{L^{6 / 5}\left(M \backslash \Omega_{L}\right)}+\left\|\rho\left(|\psi|^{2}-1\right)\right\|_{L^{6 / 5}\left(\Omega_{L}\right)}+\|\rho\|_{L^{6 / 5}\left(\Omega_{L}\right)}\right) \\
& \leq \frac{36}{k^{2}}\left((L+1)\|\rho\|_{\frac{6}{5}}+\|\rho\|_{L^{3 / 2}\left(\Omega_{L}\right)}\left\||\psi|^{2}-1\right\|_{L^{6}\left(\Omega_{L}\right)}\right) .
\end{aligned}
$$

We collect all the terms which involve $\left\||\psi|^{2}-1\right\|_{L^{6}\left(\Omega_{c}\right)}$,

$$
\left(1-\frac{36}{k^{2}}\|\rho\|_{L^{3 / 2}\left(\Omega_{L}\right)}\right)\left\||\psi|^{2}-1\right\|_{L^{6}\left(\Omega_{L}\right)} \leq \frac{36}{k^{2}}(L+1)\|\rho\|_{\frac{6}{5}} .
$$

This inequality gives a bound for $\left\||\psi|^{2}-1\right\|_{L^{6}\left(\Omega_{L}\right)}$ only if the prefactor is bounded away from zero. Thus we want to arrange that

$$
\frac{36}{k^{2}}\|\rho\|_{L^{3 / 2}\left(\Omega_{L}\right)} \leq \frac{1}{2} .
$$

The Hölder inequality gives

$$
\|\rho\|_{L^{3 / 2}\left(\Omega_{L}\right)} \leq\|\rho\|_{3} \mu\left(\Omega_{L}\right)^{\frac{1}{3}} .
$$

Substituting in the volume bound of Lemma 3.1. one sees that (3.6) indeed guarantees that (3.9) holds.

\section{Estimates of the Spinor Operator}

We choose an orthonormal basis of constant spinors $\left(\psi_{0}^{i}\right)_{i=1, \ldots, 4},\left(\psi_{0}^{i}, \psi_{0}^{j}\right) \equiv \delta_{i j}$ at the asymptotic end and denote the corresponding solutions of the boundary problems (2.5) by $\left(\psi^{i}\right)_{i=1, \ldots, 4}$. 
For every $x \in M$ we introduce the spinor operator $\Pi_{x}$ by

$$
\Pi_{x}: \Sigma_{N, x} \rightarrow \Sigma_{N, x}, \psi \mapsto \sum_{i=1}^{4}\left(\psi^{i}(x), \psi\right) \psi^{i}(x) .
$$

At infinity, $\left(\psi^{i}\right)$ goes over to an orthonormal basis, and thus

$$
\lim _{|x| \rightarrow \infty} \Pi_{x}=\mathrm{id}
$$

The next elementary lemma bounds the spinor operator in terms of the $\left|\psi^{i}\right|$.

Lemma 4.1 The sup-norm of $\Pi_{x}$ is bounded by

$$
\frac{1}{4} \sum_{j=1}^{4}\left|\psi_{x}^{i}\right|^{2} \leq\left|\Pi_{x}\right| \leq \sum_{j=1}^{4}\left|\psi_{x}^{i}\right|^{2} .
$$

Proof. Since $\Pi_{x}$ is positive,

$$
\left|\Pi_{x}\right| \geq \frac{1}{4} \operatorname{tr} \Pi_{x}=\frac{1}{4} \sum_{j=1}^{4}\left|\psi_{x}^{i}\right|^{2} .
$$

This is the lower bound.

In order to derive the upper bound, we define the matrix $A$ by $A=\left(a_{i j}\right)_{\substack{i=1, \ldots, 4 \\ j=1, \ldots, 4}}$ with

$$
a_{i j}=\left(\psi_{x}^{i}, \psi_{x}^{j}\right)
$$

By definition, $A$ is Hermitian and all eigenvalues of $A$ are real and nonnegative. Let $v:=\left(v_{1}, \ldots, v_{4}\right)^{T} \in \mathbb{C}^{4},|v|^{2}=1$. Then $\psi:=\Sigma v_{i} \psi^{i}$ is a solution of the boundary problem (2.5) with $\psi_{0}=\Sigma v_{i} \psi_{0}^{i}$. Then

$$
\sum_{i, j=1}^{4} \bar{v}_{i} v_{j} a_{i j}=\left|\psi_{x}\right|^{2} \leq \sum_{j=1}^{4}\left|\psi_{x}^{i}\right|^{2}=: \lambda .
$$

Therefore the eigenvalues of $A$ must be smaller or equal to $\lambda$.

Now let $\phi$ be an arbitrary spinor at $x \in M$. We let $\psi:=\Sigma v_{i} \psi_{x}^{i}$ be the orthonormal projection of $\phi$ onto the span of $\left(\psi_{x}^{1}, \ldots \psi_{x}^{4}\right)$ and set $v^{T}=\left(v_{1}, \ldots, v_{4}\right)$. Then

$$
\begin{aligned}
\left|\Pi_{x} \phi\right|^{2} & =\sum_{i, j=1}^{4}\left(\phi, \psi_{x}^{i}\right)\left(\psi_{x}^{i}, \psi_{x}^{j}\right)\left(\psi_{x}^{j}, \phi\right)=(\overline{A v})^{T} A(A v) \\
& \leq \lambda^{2} T \bar{v} A v=\lambda^{2}\left|\psi_{x}\right|^{2} \leq \lambda^{2}|\phi|^{2}
\end{aligned}
$$

and thus $\left|\Pi_{x} \phi\right| \leq \lambda|\phi|$.

Next we derive an estimate for the Hilbert-Schmidt Norm $\|\cdot\|$ of the operator $\left\|1-\Pi_{x}\right\|$. 
Lemma 4.2 For every $L \geq 3$ and $\varepsilon \in(0,1)$ there is a subset $U \subset M$ with

$$
\mu(U)^{\frac{1}{3}} \leq \frac{48}{k^{2}}\left(4 \pi E+\|h\|_{2}^{2}\right) \frac{L^{2}\left(4+L^{2}\right)^{2}}{\varepsilon^{2}}
$$

such that for all $x \in M \backslash U$,

$$
\left\|1-\Pi_{x}\right\|<\varepsilon .
$$

Proof. We set $p(x)=\left\|1-\Pi_{x}\right\|^{2}$. Then the same calculation as in [5, Lemma 4.2], shows that

$$
p(x)=4-2 \sum_{i=1}^{4}\left(\psi_{x}^{i}, \psi_{x}^{i}\right)+\sum_{i, j=1}^{4}\left|\left(\psi_{x}^{i}, \psi_{x}^{j}\right)\right|^{2} .
$$

Differentiation gives

$$
\begin{aligned}
\nabla p= & -4 \sum_{i=1}^{4} \operatorname{Re}\left(\nabla \psi^{i}, \psi^{i}\right)+4 \sum_{i=1}^{4} \operatorname{Re}\left(\nabla \psi^{i}, \Pi \psi^{i}\right) \\
& -2 \sum_{i=1}^{4}\left(\psi^{i}, \nu \cdot \nabla \nu \cdot \psi^{i}\right)+2 \sum_{i=1}^{4}\left(\psi^{i}, \Pi\left(\nu \cdot \nabla \nu \cdot \psi^{i}\right)\right) .
\end{aligned}
$$

We define the function $\hat{p}$ by truncating $p$,

$$
\hat{p}=\min \left(p,\left(\frac{L^{2}}{4}-2\right)^{2}\right) .
$$

Then $\nabla \hat{p}(x)$ vanishes unless $p(x) \leq\left(\frac{L^{2}}{4}-2\right)^{2}$. In this case, we have

$$
\left(\frac{L^{2}}{4}-2\right)^{2} \geq p(x) \geq\left(\left\|\Pi_{x}\right\|-\|1\|\right)^{2}
$$

and thus $\left\|\Pi_{x}\right\| \leq \frac{L^{2}}{4}$. According to Lemma 4.1, this implies that $\left|\psi^{i}\right| \leq L$ for all $i=$ $1, \ldots, 4$. We conclude that

$$
\nabla \hat{p}(x) \neq 0 \quad \Longrightarrow \quad\left|\psi^{i}(x)\right| \leq L .
$$

The last inequality allows us to estimate $\nabla \hat{p}$ as follows,

$$
|\nabla \hat{p}| \leq 4 L \sum_{i=1}^{4}\left|\nabla \psi^{i}\right|+L^{3} \sum_{i=1}^{4}\left|\nabla \psi^{i}\right|+8 L^{2}|h|+2 L^{4}|h|
$$

with $|h|^{2}=\sum_{i, k} h_{i k}^{2}$. Integration gives

$$
\begin{aligned}
\|\nabla \hat{p}\|_{2}^{2} & \leq 2 L^{2}\left(4+L^{2}\right)^{2}\left(\sum_{i=1}^{4}\left\|\nabla \psi^{i}\right\|_{2}^{2}+4 L^{2}\|h\|_{2}^{2}\right) \\
& =8 L^{2}\left(4+L^{2}\right)^{2}\left(4 \pi E+\|h\|_{2}^{2}\right) .
\end{aligned}
$$

The Sobolev inequality yields

$$
\|\hat{p}\|_{6}^{2} \leq \frac{48 L^{2}\left(4+L^{2}\right)^{2}}{k^{2}}\left(4 \pi E+\|h\|_{2}^{2}\right) .
$$

Hence $\hat{p}(x)<\varepsilon$ except for $x \in U$, where the measure of $U$ is bounded by

$$
\mu(U)^{1 / 3} \leq \frac{48 L^{2}\left(4+L^{2}\right)^{2}}{\varepsilon^{2} k^{2}}\left(4 \pi E+\|h\|_{2}^{2}\right) .
$$

Clearly, on $M \backslash U$, also $p(x)<\varepsilon$. 


\section{Estimates of the Curvature Tensor}

We denote the curvature tensor of $\Sigma_{N}$ restricted to $M$ by $\bar{R}_{M}^{\Sigma}:=i^{*} \bar{R}^{\Sigma} \in \Omega^{2}\left(M, \operatorname{End}\left(\Sigma_{N}\right)\right)$, where $i$ is the natural inclusion $i: M \rightarrow N$. Recall that $\bar{R}^{\Sigma}$ is related to the Riemannian curvature tensor $\bar{R}$ by (2.2). We denote the pull-back of $\bar{R}$ to $M$ by $\bar{R}_{M}$ and define its norm by

$$
\left|\bar{R}_{M}\right|^{2}=\sum_{i, j=1}^{3} \sum_{\alpha, \beta=0}^{3}\left(\bar{R}_{i j \alpha \beta}\right)^{2}
$$

We now derive a pointwise estimate for the curvature tensor in terms of the system of Dirac spinors $\psi^{i}$.

\section{Lemma 5.1}

$$
(1-\|1-\Pi\|)\left|\bar{R}_{M}\right|^{2} \leq 8 \sum_{i=1}^{4}\left|\bar{\nabla}^{2} \psi^{i}\right|_{\otimes 2}^{2} .
$$

Proof. The identity $\bar{R}_{M}^{\Sigma}(v, w) \psi=\bar{\nabla}^{2} \psi(v, w)-\bar{\nabla}^{2} \psi(w, v)$ immediately yields that

$$
\left|\bar{R}_{M}^{\Sigma} \psi\right|_{\otimes 2}^{2} \leq 4\left|\bar{\nabla}^{2} \psi\right|_{\otimes 2}^{2}
$$

In order to estimate the term on the left, we choose for given $x \in M$ an orthonormal frame $\left(\nu, e_{1}, e_{2}, e_{3}\right)$ with $\bar{\nabla} e_{i}(x)=0$ and an orthonormal basis $\left(\phi_{a}\right)_{a=1, \ldots, 4}$ of $\Sigma_{N, x}$. Then for any linear map $A \in \operatorname{End}\left(\Sigma_{N, x}\right)$,

$$
\operatorname{Tr}(A \Pi)(x)=\sum_{a=1}^{4}\left(\phi_{a}, A \Pi_{x} \phi_{a}\right)(x)=\sum_{i=1}^{4}\left(\psi_{i}(x), A \psi_{i}(x)\right) .
$$

Thus

$$
\begin{aligned}
& \sum_{i=1}^{4}\left|\bar{R}_{M}^{\Sigma} \psi^{i}\right|^{2}=\sum_{i=1}^{4} \sum_{j, k=1}^{3}\left(\psi^{i}, \bar{R}_{M}^{\Sigma *}\left(e_{j}, e_{k}\right) \bar{R}_{M}^{\Sigma}\left(e_{j}, e_{k}\right) \psi^{i}\right) \\
& \quad=\sum_{j, k=1}^{3} \operatorname{Tr}\left(\bar{R}_{M}^{\Sigma *}\left(e_{j}, e_{k}\right) \bar{R}_{M}^{\Sigma}\left(e_{j}, e_{k}\right) \Pi\right) \\
& \quad \geq \sum_{j, k=1}^{3}\left(\left\|\bar{R}_{M}^{\Sigma}\left(e_{j}, e_{k}\right)\right\|^{2}-\left\|\bar{R}_{M}^{\Sigma}\left(e_{j}, e_{k}\right) \bar{R}_{M}^{\Sigma *}\left(e_{j}, e_{k}\right)\right\|\|1-\Pi\|\right) .
\end{aligned}
$$

Next we compute the appearing Hilbert-Schmidt norms.

$$
\begin{aligned}
\bar{R}_{M}^{\Sigma *}\left(e_{i}, e_{j}\right) & \bar{R}_{M}^{\Sigma}\left(e_{i}, e_{j}\right) \\
= & \frac{1}{16}\left(-\sum_{k, l, m, n=1}^{3} \bar{R}_{i j k l} \bar{R}_{i j m n} e_{k} \cdot e_{l} \cdot e_{m} \cdot e_{n}+2 \sum_{k, l, m=1}^{3} \bar{R}_{i j 0 k} \bar{R}_{i j l m} \nu \cdot e_{k} \cdot e_{l} \cdot e_{m}\right. \\
& \left.\quad+2 \sum_{k, l, m=1}^{3} \bar{R}_{i j k l} \bar{R}_{i j 0 m} e_{k} \cdot e_{l} \cdot \nu \cdot e_{m}+4 \sum_{k, l=1}^{3} \bar{R}_{i j o k} \bar{R}_{i j 0 m} \nu \cdot e_{k} \cdot \nu \cdot e_{m}\right) \\
= & \frac{1}{8}\left(\sum_{k, l=1}^{3} \bar{R}_{i j k l} \bar{R}_{i j k l}+2 \sum_{k, l, m=1}^{3} \bar{R}_{i j 0 k} \bar{R}_{i j l m} \nu \cdot e_{k} \cdot e_{l} \cdot e_{m}+2 \sum_{k=1}^{3} \bar{R}_{i j 0 k} \bar{R}_{i j 0 k}\right)
\end{aligned}
$$


Since the trace of the second term vanishes, we conclude that

$$
\sum_{j, k=1}^{3}\left\|\bar{R}_{M}^{\Sigma}\left(e_{j}, e_{k}\right)\right\|^{2}=\frac{1}{2}\left|\bar{R}_{M}\right|^{2} .
$$

Moreover,

$$
\sum_{j, k=1}^{3}\left\|\bar{R}_{M}^{\Sigma}\left(e_{j}, e_{k}\right) \bar{R}_{M}^{\Sigma *}\left(e_{j}, e_{k}\right)\right\| \leq \sum_{j, k=1}^{3}\left\|\bar{R}_{M}^{\Sigma}\left(e_{j}, e_{k}\right)\right\|^{2} \leq \frac{1}{2}\left|\bar{R}_{M}\right|^{2},
$$

Substituting (5.4) and (5.5) into (5.3) gives the result.

\section{Integration by Parts}

In this section we derive an $L^{2}$ bound for the second derivative of a solution of the boundary value problem (2.5). The argument is similar to that given in [4].

Lemma 6.1 Suppose that L satisfies the hypothesis of Proposition 3.3. Then any solution $\Psi$ of the boundary value problem (2.5) satisfies the inequality

$$
\begin{aligned}
& \int_{M} \eta\left|\nabla^{2} \psi\right|^{2} d \mu \leq c_{1} \sup _{M}\left(|\Delta \eta|+|\nabla \eta||h|+\eta\left(|R|+|h|^{2}+|\bar{\nabla} h|\right) E\right. \\
& \quad+c_{2} L \sup _{M}\left(\eta\left(\left|\overline{\nabla R}_{M}\right|+|h|\left|\bar{R}_{M}\right|\right)\right) \sqrt{E} \\
& \quad+c_{3} \frac{\sqrt{L+1}}{k}\left(\sup _{M} \eta\right) \sqrt{\left\||h|^{2}+|\nabla h|\right\|_{6 / 5}}\left\|\left|\overline{\nabla R}_{M}\right|+|h| \mid \bar{R}_{M}\right\|_{5 / 12} \sqrt{E}
\end{aligned}
$$

Proof. A calculation similar to the one following (3.5) yields that

$$
\begin{aligned}
\left|\bar{\nabla}^{2} \psi\right|^{2}= & \sum_{j, i} \operatorname{Re}\left(\bar{\nabla}_{j}^{*} \bar{\nabla}_{j, i}^{2} \psi, \bar{\nabla}_{i} \psi\right) \\
& +\frac{1}{2} \Delta|\bar{\nabla} \psi|^{2} \\
& -\frac{1}{2} \sum_{j, i}\left(\bar{\nabla}_{i} \psi, \nu \cdot \bar{\nabla}_{j, j}^{2} \nu \cdot \bar{\nabla}_{i} \psi\right) \\
& -\sum_{j, i} \operatorname{Re}\left(\bar{\nabla}_{j, i}^{2} \psi, \nu \cdot \bar{\nabla}_{j} \nu \cdot \bar{\nabla}_{i} \psi\right),
\end{aligned}
$$

where $\left(e_{1}, \ldots, e_{n}\right)$ is a smooth orthonormal frame on $M$.

In order to estimate the integral $\int_{M} \eta\left|\bar{\nabla}^{2} \psi\right| d \mu$ with a positive test function $\eta \in C^{2}(M)$,we consider the summands in the above equation separately. Integrating by parts in (6.2) and using the decay properties of $\psi$, we obtain

$$
\frac{1}{2} \int_{M} \eta \Delta|\bar{\nabla} \psi|^{2} d \mu=\frac{1}{2} \int_{M} \Delta \eta|\bar{\nabla} \psi|^{2} d \mu \leq 4 \pi \sup _{M} \Delta \eta E .
$$


To estimate (6.3) and (6.4), we first calculate

$$
\begin{aligned}
& \frac{1}{2} \bar{\nabla}_{j}\left(\bar{\nabla}_{i} \psi, \nu \cdot \bar{\nabla}_{j} \nu \cdot \bar{\nabla}_{i} \psi\right)=\frac{1}{2} \bar{\nabla}_{j}\left\langle\bar{\nabla}_{i} \psi, \bar{\nabla}_{j} \nu \cdot \bar{\nabla}_{i} \psi\right\rangle \\
& \quad=\operatorname{Re}\left(\bar{\nabla}_{j, i}^{2} \psi, \nu \cdot \bar{\nabla}_{j} \nu \cdot \bar{\nabla}_{i} \psi\right)+\frac{1}{2}\left(\bar{\nabla}_{i} \psi, \nu \cdot \bar{\nabla}_{j, j}^{2} \nu \cdot \bar{\nabla}_{i} \psi\right) .
\end{aligned}
$$

Therefore, integration by parts gives

$$
\begin{aligned}
& \left|\sum_{i, j} \int_{M} \eta\left(\frac{1}{2}\left(\bar{\nabla}_{i} \psi, \nu \cdot \bar{\nabla}_{j, j}^{2} \nu \cdot \bar{\nabla}_{i} \psi\right)+\operatorname{Re}\left(\bar{\nabla}_{j, i}^{2} \psi, \nu \cdot \bar{\nabla}_{j} \nu \cdot \bar{\nabla}_{i} \psi\right)\right) d \mu\right| \\
& \leq \frac{1}{2} \sum_{i, j} \int_{M}\left|\left(\partial_{j} \eta\right)\left(\bar{\nabla}_{i} \psi, \nu \cdot \bar{\nabla}_{j} \nu \cdot \bar{\nabla}_{i} \psi\right)\right| d \mu \leq 4 \pi \sup _{M}(|\nabla \eta||h|) E .
\end{aligned}
$$

It remains to control (6.1). Commuting the covariant derivatives, we obtain, as in [4, eqns. (31)-(35)],

$$
\begin{aligned}
\bar{\nabla}_{j}^{*} \bar{\nabla}_{j, i}^{2} \psi= & \bar{\nabla}_{i}\left(\bar{\nabla}^{*} \bar{\nabla} \psi\right)+\frac{1}{2} \bar{\nabla}_{j}^{*}\left(\bar{R}^{\Sigma}\left(e_{j}, e_{i}\right) \psi\right)+ \\
& +\operatorname{Ric}\left(e_{i}, e_{k}\right) \bar{\nabla}_{k} \psi-\frac{1}{2} \bar{R}^{\Sigma}\left(e_{j}, e_{j}\right) \bar{\nabla}_{j} \psi+\nu \cdot \bar{\nabla}_{i, j}^{2} \nu \cdot \bar{\nabla}_{j} \psi,
\end{aligned}
$$

where Ric denotes the Ricci curvature of the hypersurface $M \subset N$. If $\psi$ is a solution of (2.5), the first term can be simplified with the Weitzenböck formula. Using the Gauss equation, we thus obtain

$$
\left(\bar{\nabla}_{j}^{*} \bar{\nabla}_{j, i}^{2} \psi, \bar{\nabla}_{i} \psi\right) \leq \tilde{c}_{1}\left(|R|+|h|^{2}+|\bar{\nabla} h|\right)|\bar{\nabla} \psi|^{2}+\tilde{c}_{2}\left(\left|\overline{\nabla R}_{i}\right|+|h|\left|\bar{R}_{i}\right|\right)\left(\psi, \bar{\nabla}_{i} \psi\right)
$$

with suitable constants $\tilde{c}_{1}$ and $\tilde{c}_{2}$ which are independent of the geometry. Now we choose $L>0$ as in Proposition 3.3 and calculate

$$
\begin{aligned}
\int_{M} \eta \operatorname{Re}\left(\bar{\nabla}_{j}^{*} \bar{\nabla}_{j, i}^{2} \psi, \bar{\nabla}_{i} \psi\right) d \mu \leq \tilde{c}_{3} \sup _{M}\left(\eta\left(|R|+|h|^{2}+|\bar{\nabla} h|\right) E\right. \\
\quad+\tilde{c}_{2}\left(\int_{M \backslash \Omega_{L}}+\int_{\Omega_{L}}\right) \eta\left(\left|\bar{\nabla}^{*} \bar{R}_{M}\right|+|h|\left|\bar{R}_{M}\right|\right)|\psi||\bar{\nabla} \psi| d \mu \\
\leq \quad \tilde{c}_{3} \sup _{M}\left(\eta\left(|R|+|h|^{2}+|\bar{\nabla} h|\right) E\right. \\
\left.\quad+\tilde{c}_{4} L \sup _{M}\left(\eta\left(\mid \bar{\nabla} \bar{R}_{M}\right)|+| h|| \bar{R}_{M} \mid\right)\right) \sqrt{E} \\
\quad+\tilde{c}_{5} \int_{\Omega_{L}} \eta\left(\left|\bar{\nabla} \bar{R}_{M}\right|+|h|\left|\bar{R}_{M}\right|\right) \sqrt{|\psi|^{2}-1}|\bar{\nabla} \psi| d \mu
\end{aligned}
$$

where we have used the inequality $|\psi| \leq \sqrt{|\psi|^{2}-1}+1$. In the last integral, we apply Hölder's inequality,

$$
\begin{aligned}
& \int_{\Omega_{L}} \eta\left(\left|\bar{\nabla} \bar{R}_{M}\right|+|h|\left|\bar{R}_{M}\right|\right) \sqrt{|\psi|^{2}-1}|\bar{\nabla} \psi| d \mu \\
& \quad \leq \sup _{M} \eta\left\|\left|\bar{\nabla} \bar{R}_{M}\right|+|h|\left|\bar{R}_{M}\right|\right\|_{\frac{5}{12}}\left\||\psi|^{2}-1\right\|_{L^{6}\left(\Omega_{L}\right)}^{\frac{1}{2}}\|\nabla \psi\|_{2} .
\end{aligned}
$$


Finally, the factor $\left\||\psi|^{2}-1\right\|_{L^{6}\left(\Omega_{L}\right)}$ is controlled by Proposition 3.3.

Proof of Theorem 1.3. For $L$ as in Proposition 3.3 and $\varepsilon=\frac{1}{2}$, we choose $U$ as in Lemma 4.2 to obtain

$$
\int_{M \backslash U} \eta\left|\bar{R}_{M}\right| d \mu \leq 2 \int_{M \backslash U} \eta(1-\|1-\Pi\|)\left|\bar{R}_{M}\right| d \mu .
$$

We now apply Lemma 5.1,

$$
\int_{M \backslash U} \eta\left|\bar{R}_{M}\right| d \mu \leq 16 \int_{M} \eta \sum_{i=1}^{4}\left|\bar{\nabla}^{2} \psi_{i}\right|_{\otimes 2}^{2} d \mu .
$$

Lemma 6.1 completes the proof.

\section{References}

[1] R. Arnowitt, S. Deser, C. Misner, "Energy and the criteria for radiation in general relativity," Phys. Rev. 118, 1100 (1960)

[2] R. Bartnik, "The Mass of an Asymptotically Flat Manifold", Commun. Pure Appl. Math. XXXIX, 661-693 (1986)

[3] H. Baum, "Spin-Strukturen und Dirac-Operatoren über pseudo-Riemannschen Mannigfaltigkeiten," Teubner Verlag Leipzig (1981)

[4] H. Bray, F. Finster, "Curvature estimates and the positive mass theorem," Comm. Anal. Geom. 10 (2002) 291-306

[5] F. Finster, I. Kath, "Curvature estimates in asymptotically flat manifolds of positive scalar curvature," Comm. Anal. Geom. 10 (2002) 1017-1031

[6] H.-B. Lawson, M.-L. Michelsohn, Spin Geometry, Princeton University Press, Princeton 1989.

[7] R. Schoen, S.-T. Yau, "On the proof of the positive mass conjecture in general relativity," Commun. Math. Phys. 65, 45-76 (1976)

[8] T. Parker, C. H. Taubes, "On Witten's proof of the positive energy theorem," Commun. Math. Phys. 84, 223-238 (1982)

[9] E. Witten, "A new proof of the positive energy theorem," Commun. Math. Phys. 80, 381-402 (1981) 
Felix Finster

Naturwissenschaftliche Fakultät I - Mathematik

Universität Regensburg

D-93040 Regensburg

GERMANY

felix.finster@mathematik. uni-regensburg.de

Margarita Kraus

Naturwissenschaftliche Fakultät I - Mathematik

Universität Regensburg

D-93040 Regensburg

GERMANY

margarita.kraus@mathematik. uni-regensburg. de 